Article

\title{
A Model of Nonsingular Universe
}

\section{Changjun Gao ${ }^{1,2}$}

${ }^{1}$ The National Astronomical Observatories, Chinese Academy of Sciences, Beijing 100012, China; E-Mail: gaocj@bao.ac.cn

${ }^{2}$ Kavli Institute for Theoretical Physics China, CAS, Beijing 100190, China

Received: 19 April 2012; in revised form: 4 June 2012 / Accepted: 13 June 2012 /

Published: 23 July 2012

\begin{abstract}
In the background of Friedmann-Robertson-Walker Universe, there exists Hawking radiation which comes from the cosmic apparent horizon due to quantum effect. Although the Hawking radiation on the late time evolution of the universe could be safely neglected, it plays an important role in the very early stage of the universe. In view of this point, we identify the temperature in the scalar field potential with the Hawking temperature of cosmic apparent horizon. Then we find a nonsingular universe sourced by the temperature-dependent scalar field. We find that the universe could be created from a de Sitter phase which has the Planck energy density. Thus the Big-Bang singularity is avoided.
\end{abstract}

Keywords: Hawking temperature; scalar field; nonsingular universe

\section{Introduction}

The standard model of cosmology claims that the primordial Universe was in a hot, dense, and highly curved state which is very close to the Big-Bang singularity [1]. The cosmic energy density, the temperature and other physical quantities become infinite at the singularity. Thus the presence of infinity of physical quantities indicates that the Einstein's General Relativity breaks down at the singularity. General Relativity must be modified at high densities. In other words, some other aspects of physics should be taken into account in the gravity theory.

It is usually believed that the Big-Bang singularity might be regularized in the quantum version of gravity theory. Actually, the absence of the Big-Bang singularity in a quantum setting could be expected on qualitative grounds although a completely self-consistent quantum theory of gravity is still not yet available so far. As is well known, there are only four fundamental constants in the theories 
describing space, time, matter and thermodynamics. They are the Newton's constant $G$ (related to General Relativity), the speed of light $c$ (related to Special Relativity), the Planck's constant $\hbar$ (related to quantum mechanics) and the Boltzmann's constant $k$ (related to thermodynamics). Consequently, one could have only four quantities that can be constructed from these four fundamental constants. They are Planck time $t_{p}$, Planck length $l_{p}$, Planck density $\rho_{p}$ and Planck temperature $T_{p}$ [2],

$$
\begin{aligned}
t_{p} & =\sqrt{\frac{\hbar G}{c^{5}}}=5.4 \times 10^{-44} \mathrm{~s} \\
l_{p} & =\sqrt{\frac{\hbar G}{c^{3}}}=1.6 \times 10^{-35} \mathrm{~m} \\
\rho_{p} & =\frac{c^{5}}{\hbar G^{2}}=5.2 \times 10^{96} \mathrm{~kg} \cdot \mathrm{m}^{-3} \\
T_{p} & =\frac{l_{p} c^{4}}{G k}=1.4 \times 10^{32} \mathrm{~K}
\end{aligned}
$$

These quantities are expected to play important role in the quantum theory of gravity. It is generally assumed that they set the scale for the quantum gravity effects. The time, length, energy density and temperature beyond these scales are highly impossible. In view of this point, the quantum effect should be considered in General Relativity and the Universe should not be singular.

The first attempt of taking quantum effect into account in gravity theory is the elegant work of Hawking [3]. According to General Relativity, the temperature of a black hole is absolutely zero. This violates the third law of thermodynamics which states that one can never approach the temperature of zero with finite operations. However, Hawking found that a black hole behaves like a black body, emitting thermal radiation, with a temperature inversely proportional to its mass $M$,

$$
T_{H}=\frac{1}{8 \pi M} \cdot \frac{\hbar c^{3}}{G k}
$$

In the formulae, four theories of physics, Special Relativity $(c)$, General Relativity $(G)$, quantum mechanics $(\hbar)$ and thermodynamics $(k)$ are present. Then the third law of thermodynamics is not violated. Motivated by this point, one expect the Big-Bang singularity may be eliminated by taking into account the quantum effect. On the other hand, in the background of Friedmann-Robertson-Walker (FRW) Universe, it is also found that the universe is filled with the Hawking radiation which comes from the apparent horizon due to quantum effect. The temperature of Hawking radiation is give by [4-7]

$$
T_{H}=\frac{1}{2 \pi r_{A}} \cdot \frac{\hbar c}{k}
$$

where $r_{A}$ is the radius of cosmic apparent horizon. This temperature could be observed by the comoving observers in the universe. We see that not only Special relativity and thermodynamics but also quantum mechanics are present in the formulae.

The purpose of this paper is to investigate whether the Big-Bang singularity could be smoothed by simply taking account of the cosmic Hawking radiation. The answer is yes. We find that the universe could be created from a de Sitter phase which has the Planck energy density. Thus the Big-Bang singularity is avoided.

Actually, many solutions of nonsingular universe have been presented in the literature. These solutions are based on various approaches to quantum gravity such as modified gravity models [8-11], 
Lagrangian multiplier gravity actions (see e.g., [12-14]), non-relativistic gravitational actions [15-17], brane world scenarios $[18,19]$ an so on. Here we shall not produce an exhaustive list of references, but we prefer the readers to read the nice review paper by Novello and Bergliaffa [20] and the references therein.

The paper is organized as follows. In Section 2, we shall derive the Friedmann equation and the acceleration equation sourced by the temperature-dependent scalar field. In Section 3, we present a nonsingular universe by simply considering the scalar potential for the oscillators. Section 4 gives the conclusion and discussion. In the following, we shall use the system of units in which $G=c=\hbar=$ $k=1$ and the metric signature $(-,+,+,+)$ until the end of the paper.

\section{Temperature-dependent Scalar Field}

We consider the Lagrangian density as follows

$$
L=\frac{1}{2} \nabla_{\mu} \phi \nabla^{\mu} \phi+V(\phi, T)
$$

where $V(\phi, T)$ is the temperature-dependent scalar potential. The theory has been widely studied in the phase transitions of very early universe [21]. In the background of Friedmann-Robertson-Walker (FRW) Universe, $T$ could be taken as the temperature of cosmic microwave background (CMB) radiation. The evolution of the temperature is given by

$$
T_{C M B} \propto \frac{1}{a}
$$

where $a$ is the scale factor of the universe. The observations of CMB gives the present-day cosmic temperature [22]

$$
T_{C M B} \simeq 2.73 \mathrm{~K}
$$

On the other hand, in the background of the spatially-flat FRW Universe, it is found that the universe is filled with the Hawking radiation which comes from the apparent horizon due to quantum effect. The temperature of Hawking radiation is given by [4-7]

$$
T_{H}=\frac{H}{2 \pi}
$$

where $H$ is the Hubble parameter. This temperature could be observed by the comoving observers in the universe. For the present-day universe, it is approximately

$$
T_{H} \simeq 10^{-29} \mathrm{~K}
$$

It is very much smaller than the temperature of $\mathrm{CMB}$. So it is very safe to neglect the quantum effect for the present-day Universe. Using the Friedmann equation, the temperature of CMB at the radiation dominated epoch can be rewritten as

$$
T_{C M B} \propto \sqrt{H}
$$

Thus, with the increasing of redshifts, the Hawking temperature $T_{H}$ increases faster than the CMB temperature $T_{C M B}$. In other words, the quantum effect would become significant in the very early universe. In particular, the Hawking temperature at the Planck time may be as high as

$$
T_{H} \simeq 10^{32} \mathrm{~K}
$$


Since the Hawking temperature becomes very important in the very early universe, we identify the temperature in the Lagrangian, Equation (4), with not the CMB temperature but the Hawking temperature.

Taking into account gravity, we have the action as follows

$$
S=\int d^{4} x \sqrt{-g}\left[\frac{R}{16 \pi}+\frac{1}{2} \nabla_{\mu} \phi \nabla^{\mu} \phi+V(\phi, T)\right]
$$

where $R$ is the Ricci scalar. The metric for the spatially flat FRW Universe is given by

$$
d s^{2}=-d t^{2}+a(t)^{2}\left(d r^{2}+r^{2} d \theta^{2}+r^{2} \sin ^{2} \theta d \varphi^{2}\right)
$$

where $a(t)$ is the scale factor of the Universe. Then the action becomes

$$
\begin{aligned}
S= & \int 4 \pi r^{2} d r \int d t\left[\frac{1}{16 \pi}\left(-6 \ddot{a} a^{2}-6 \dot{a}^{2} a\right)\right. \\
& \left.-\frac{1}{2} a^{3} \dot{\phi}^{2}+a^{3} V(\phi, H)\right]
\end{aligned}
$$

where dot denotes the derivative with respect to cosmic time $t$. Here we have replaced the Hawking temperature with the Hubble parameter in the scalar potential. Variation of the action with respect to the scale factor $a$ gives the acceleration equation

$$
2 \dot{H}+3 H^{2}=-8 \pi\left[\frac{1}{2} \dot{\phi}^{2}-V+H \frac{\partial V}{\partial H}+\frac{1}{3}\left(\frac{\partial V}{\partial H}\right)\right]
$$

On the other hand, variation of the action with respect to the scalar field $\phi$ gives the equation of motion for $\phi$

$$
\ddot{\phi}+3 H \dot{\phi}+\frac{\partial V}{\partial \phi}=0
$$

Equation (14) tells us the scalar field contributes a pressure as the following

$$
p=\frac{1}{2} \dot{\phi}^{2}-V+H \frac{\partial V}{\partial H}+\frac{1}{3}\left(\frac{\partial V}{\partial H}\right)
$$

Compared to the pressure of quintessence,

$$
p=\frac{1}{2} \dot{\phi}^{2}-V
$$

the last two terms on the right hand side of Equation (16) come from the variation of scalar potential with respect to the Hawking temperature.

In order to find the energy density $\rho$ contributed by the scalar field, we put

$$
\rho=\frac{1}{2} \dot{\phi}^{2}+V+F
$$

where $F$ is a function to be determined. Substituting Equation (16) and Equation (18) into the energy conservation equation

$$
\dot{\rho}+3 H(\rho+p)=0
$$


and taking into account Equation (15), we obtain

$$
\left(F+H \frac{\partial V}{\partial H}\right)+3 H\left(F+H \frac{\partial V}{\partial H}\right)=0
$$

So we get

$$
F=-H \frac{\partial V}{\partial H}+\frac{F_{0}}{a^{3}}
$$

where $F_{0}$ is an integration constant. Since there is no constant in the Lagrangian density, Equation (4), we expect $F_{0}$ should be zero. So the energy density is given by

$$
\rho=\frac{1}{2} \dot{\phi}^{2}+V-H \frac{\partial V}{\partial H}
$$

The last term comes from the variation of the scalar potential with respect to the Hawking temperature.

Now we can write the Friedmann equation as the following

$$
3 H^{2}=8 \pi\left(\frac{1}{2} \dot{\phi}^{2}+V-H \frac{\partial V}{\partial H}\right)
$$

Equations (14), (15) and (23) constitute the main equations which govern the evolution of the universe. Among the three equations, only two of them are independent. But we have three unknown functions, $a(t), \phi(t)$ and $V(\phi, T)$. So we are left with one degree of freedom. For simplicity, we may fix the expression of the scalar potential.

\section{A Nonsingular Universe}

We consider one of the simplest scalar potentials given by

$$
V(\phi, T)=2 \pi^{2} \eta^{2} T^{2} \phi^{2}
$$

with $\eta$ a positive dimensionless constant. This form of temperature-dependent potential comes from the high-temperature expansion of the finite-temperature effective potential [23]:

$$
V_{T}(\phi, T)=V(\phi)+\frac{\lambda}{8} T^{2} \phi^{2}-\frac{\pi^{2}}{90} T^{4}+\cdots
$$

where $\lambda$ is a constant. For simplicity and to the first order of temperature corrections, we are only interested in the second term in the right hand of the equation.

Using the formulae of temperature, Equation (7), we can rewrite the scalar potential as follows

$$
V(\phi, T)=\frac{1}{2} \eta^{2} H^{2} \phi^{2}
$$

Now the Friedmann equation, Equation (23), is reduced to

$$
3=4 \pi\left[\left(\frac{d \phi}{d x}\right)^{2}-\eta^{2} \phi^{2}\right]
$$

where $x$ is defined by

$$
x \equiv \ln a
$$


Solving the differential equation, we find

$$
\phi=\sqrt{\frac{3}{4 \pi}} \frac{a^{\eta}-a^{-\eta}}{2 \eta}
$$

Without the loss of the generality, we set the integration constant to be zero.

Substituting it into the equation of motion, Equation (15), and using Equation (22) we obtain the energy density

$$
\rho=\frac{\rho_{p} a^{4 \eta}}{a^{6}\left(1+a^{2 \eta}\right)^{4}}
$$

where $\rho_{p}$ is the integration constant. We will shortly find that it is the Planck energy density. Substituting the energy density into the energy conservation equation, Equation (19) we obtain the equation of state $w$

$$
w \equiv \frac{p}{\rho}=1-\frac{4}{3} \eta \cdot \frac{1-a^{2 \eta}}{1+a^{2 \eta}}
$$

In order that the energy density is not divergent when $a=0$, we should require that

$$
\eta \geq \frac{3}{2}
$$

On the other hand, in order that the equation of state parameter is not less than minus one when $a=0$, we should require that

$$
\eta \leq \frac{3}{2}
$$

So $\eta$ is forced to be

$$
\eta=\frac{3}{2}
$$

Then the energy density is given by

$$
\rho=\frac{\rho_{p}}{\left(1+a^{3}\right)^{4}}
$$

So the energy density increases with the increasing of redshifts. When $a=0$, we have the maximum energy density

$$
\rho=\rho_{p}
$$

It is generally believed that the Planck energy density may be the maximum density in the universe. So the integration constant $\rho_{p}$ is actually the Planck density. The evolution of the scale factor is found to be

$$
\ln a+\frac{1}{6} a^{6}+\frac{2}{3} a^{3}=\sqrt{\frac{8 \pi}{3}} \cdot \frac{t}{t_{p}}
$$

where $t_{p}$ is the Planck time.

In Figure 1, we plot the evolution of the scale factor with respect to the cosmic time. It shows that the scale factor approaches zero when $t=-\infty$. The scale factor is actually physically meaningless. So 
we do not worry about its vanishing at $t=-\infty$. It is the radius $s$ of Hubble horizon that describes the physical size of the universe

$$
s \equiv \frac{1}{H}
$$

Figure 1. The evolution of the scale factor $a$ with respect to the cosmic time $t$. It shows that the scale factor approaches zero when $t=-\infty$. The unite of time is the Planck time $t_{p}$.

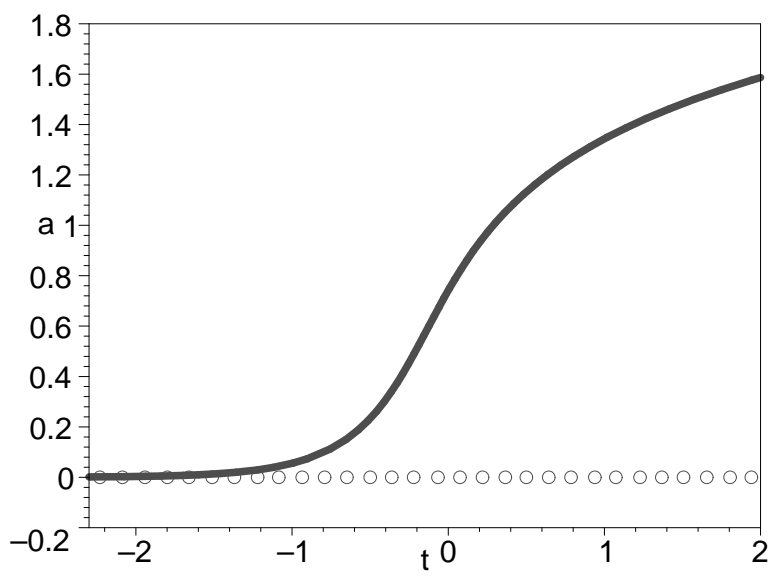

In Figure 2, we plot the evolution of the Hubble horizon $s$ with respect to the cosmic time $t$. It shows that the Hubble horizon approaches a finite constant when $t=-\infty$. Therefore, the universe is nonsingular. In Figure 3, we plot the evolution of the energy density $\rho$ with respect to the cosmic time $t$. It shows that the energy density approaches the Planck density when $t=-\infty$. Since the universe is created with the finite Hubble scale and finite energy density, it is a nonsingular universe. We note that the cosmic time $t$ is real and physical. So the minus infinity $t=-\infty$ is real and geodesic one.

Figure 2. The evolution of the Hubble radius $s$ with respect to the cosmic time $t$. It shows that the Hubble horizon approaches a finite constant when $t=-\infty$. In other words, the physical size of the universe is bounded below. The unit of time and length are the Planck time $t_{p}$ and Planck length $l_{p}$, respectively.




Figure 3. The evolution of energy density $\rho$ with respect to the cosmic time $t$. It shows that the energy density approaches the Planck density when $t=-\infty$. The unit of time and energy density are the Planck time $t_{p}$ and the Planck density $\rho_{p}$, respectively.

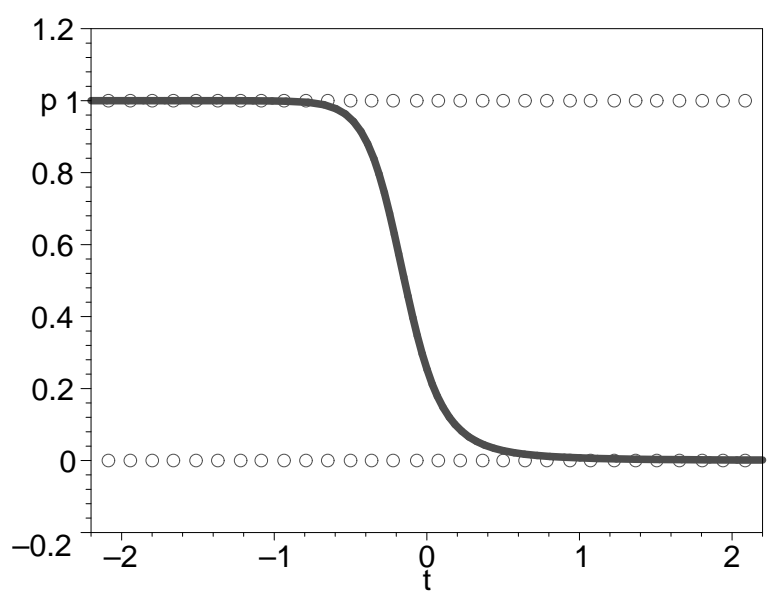

\section{Conclusion and Discussion}

Although the Hawking radiation on the late time evolution of the universe could be safely neglected, it plays an important role in the very early stage of the universe. In view of this point, we identify the temperature in the scalar field potential with the Hawking temperature of cosmic apparent horizon. Then we find a nonsingular universe sourced by the Hawking-temperature-dependent scalar field. We had better point out that the analysis of singularity here needs more attentions. For example, we need to modify gravity in a non-perturbation fashion to really make sense [24,25].

The extension of the idea to the general scalar-tensor theories is straightforward:

$$
L=f\left(R, \frac{1}{2} \nabla_{\mu} \phi \nabla^{\mu} \phi, \phi, T\right)
$$

with $f$ being an arbitrary function of the variables. But we note that the whole approach of this paper can be applied only to the FRW universe, because for a general geometry the Hawking temperature has to be defined separately. As another example, we may take $\phi$ as a constant and take the potential as follows

$$
V \propto T^{4}
$$

Then we would have the Friedmann equation as follows

$$
3 H^{2}=16 \pi \rho_{p}\left(1-\sqrt{1-\frac{\rho}{\rho_{p}}}\right)
$$

where $\rho$ may be the energy density of radiation, dark matter and dark energy. It is apparent the maximum of $\rho$ is the Planck density. It reveals a nonsingular universe. When $\rho \ll \rho_{p}$, it goes back the usual Friedmann equation.

On the other hand, if we take the potential as

$$
V \propto \frac{1}{T^{2}}
$$


we would have the Friedmann equation as follows

$$
3 H^{2}=4 \pi \rho\left(1+\sqrt{1-\frac{\rho_{X}^{2}}{\rho^{2}}}\right)
$$

where $\rho_{X}$ is some constant. It is apparent the minimum of $\rho$ is $\rho_{X}$. It reveals a nonsingular but future universe. When $\rho_{X} \leq \rho$, it restores to the usual Friedmann equation. Of course, when $T=$ const, it is the quintessence.

\section{Acknowledgements}

I thank the anonymous referees for the expert and insightful comments, which have certainly improved the paper significantly. I thank Hao Wei and Yunsong Piao for helpful discussions. This work is supported by the National Science Foundation of China under the Key Project Grant 10533010, Grant 10575004, Grant 10973014, and the 973 Project (No. 2010CB833004).

\section{References}

1. Weinberg, S. Cosmology and Gravitation; Wiley: New York, NY, USA, 1972.

2. Wald, R.M. Genreral Relativity; University of Chicago Press: Chicago, IL, USA, 1984.

3. Hawking, S.W. Particle creation by black holes. Commun. Math. Phys. 1975, 43, 199-220.

4. Medved, A.J.M. Radiation via tunneling from a de Sitter cosmological horizon. Phys. Rev. D 2002, 66, 124009.

5. Cai, R.G.; Cao, L.M.; Hu, Y.P. Hawking radiation of apparent horizon in a FRW universe. Class. Quant. Grav. 2009, 26, 155018.

6. Zhu, T.; Ren, J.-R. Corrections to Hawking-like radiation for a Friedmann-Robertson-Walker universe. Eur. Phys. J. C 2009, 62, 413-418.

7. Jang, K.-X.; Feng, T.; Peng, D.-T. Hawking radiation of apparent horizon in a FRW universe as tunneling beyond semiclassical approximation. Int. J. Theor. Phys. 2009, 48, 2112-2121.

8. Brustein, R.; Madden, R. A model of graceful exit in string cosmology. Phys. Rev. D 1998, 57, 712-724.

9. Cartier, C.; Copeland, E.J.; Madden, R. The graceful exit in pre-big bang string cosmology. J. High Energ. Phys. 2000, 0001, 035.

10. Tsujikawa, S.; Brandenberger, R.; Finelli, F. On the construction of nonsingular Pre-Big-Bang and Ekpyrotic cosmologies and the resulting density perturbations. Phys. Rev. D. 2002, 66, 1-20.

11. Biswas, T.; Mazumdar, A.; Siegel, W. Bouncing universes in string-inspired gravity. J. Cosmol. Astropart. Phys. 2006, 0603, 009.

12. Mukhanov, V.F.; Brandenberger, R.H. A nonsingular universe. Phys. Rev. Lett. 1992, 68, 1969-1972.

13. Easson, D.A.; Brandenberger, R.H. Nonsingular dilaton cosmology in the string frame. J. High Energ. Phys. 1999, 9909, 003.

14. Cai, Y.F.; Saridakis, E.N. Cyclic cosmology from Lagrange-multiplier modified gravity. Class. Quant. Grav. 2011, 28, 035010. 
15. Brandenberger, R. Matter bounce in Horava-Lifshitz cosmology. Phys. Rev. D 2009, 80, 043516.

16. Gao, X.; Wang, Y.; Xue, W.; Brandenberger, R. Fluctuations in a Horava-Lifshitz bouncing cosmology. J. Cosmol. Astropart. Phys. 2010, 1002, 020.

17. Cai, Y.F.; Saridakis, E.N. Non-singular cosmology in a model of non-relativistic gravity. J. Cosmol. Astropart. Phys. 2009, 0910, 020.

18. Kehagias, A.; Kiritsis, E. Mirage cosmology. J. High Energ. Phys. 1999, 9911, 022.

19. Shtanov, Y.; Sahni, V. Bouncing braneworlds. Phys. Lett. B 2003, 557, 1-6.

20. Novello, M.; Bergliaffa, S.E.P. Bouncing cosmologies. Phys. Rept. 2008, 463, 127-213.

21. Linde, A.D. Particle physics and inflationary cosmology. Contemp. Concepts Phys. 2005, 5, $1-362$.

22. Komatsu, E.; Dunkley, J.; Nolta, M.R.; Bennett, C.L.; Gold, B.; Hinshaw, G.; Jarosik, N.; Larson, D.; Limon, M.; Page, L.; et al. Five-year Wilkinson Microwave Anisotropy Probe (WMAP) observations: Cosmological interpretation. Astrophys. J. Suppl. 2009, 180, 330-376.

23. Kolb, E.W.; Turner, M.S. The Early Universe; AddisonWesley Publishing Company: California, CA, USA, 1990.

24. Biswas, T.; Gerwick, E.; Koivisto, T.; Mazumdar, A. Towards singularity- and ghost-free theories of gravity. Phys. Rev. Lett. 2012, 108, 1-4.

25. Biswas, T.; Gerwick, E.; Koivisto, T.; Mazumdar, A. Towards a resolution of the cosmological singularity in non-local higher derivative theories of gravity. J. Cosmol. Astropart. Phys. 2010, $1011,008$.

(c) 2012 by the author; licensee MDPI, Basel, Switzerland. This article is an open access article distributed under the terms and conditions of the Creative Commons Attribution license (http://creativecommons.org/licenses/by/3.0/). 